\title{
LESÃO RENAL GLOMERULAR: PROCESSO INFLAMATÓRIO E O PAPEL DAS QUIMIOCINAS
}

\author{
Thalles Trindade Abreu ${ }^{1}$ \\ Núbia Chouchounova Silva Neves ${ }^{1}$ \\ Lucas Ferreira Alves ${ }^{2}$ \\ Alba Otoni ${ }^{3}$
}

\begin{abstract}
ABREU, T. T.; NEVES, N. C. S.; ALVES, L. F.; OTONI, A. Lesão renal glomerular: processo inflamatório e o papel das quimiocinas. Arq. Cienc. Saúde UNIPAR, Umuarama, v. 21, n. 2, p, 131-136, maio/ago. 2017.

RESUMO: As lesões renais podem ser explicadas, de um modo geral, por agressões que causam danos às células e/ou tecidos e à resposta inflamatória gerada por esse processo a partir da ativação do sistema imune e estímulo contínuo de sua ação. Visando realizar o diagnóstico precoce da doença renal crônica e minimizar os aspectos econômicos e os impactos à vida do paciente causados pela instalação e progressão da doença renal, muitos autores procuram explicar os processos inflamatórios que são as linhas de bases de muitas doenças crônicas e os biomarcadores que participam desse processo. Este artigo tem por objetivo fazer uma revisão de literatura narrativa sobre a fisiopatologia da progressão da doença renal glomerular, bem como, as possíveis funções das quimiocinas como marcadores de progressão da doença renal.
\end{abstract}

PALAVRAS-CHAVES: Biomarcadores. Insuficiência renal crônica. Quimiocinas.

\section{GLOMERULAR KIDNEY INJURY: THE INFLAMMATORY PROCESS AND THE ROLE OF CHEMOKINES}

ABSTRACT: Kidney injuries can generally be explained as attacks damaging cells and/or tissues and the inflammatory response generated by such process due to the activation of the immune system and its continuous stimulation. Aiming to reach an early diagnosis of CKD and minimize both the economic aspects and the impacts on the patient's life caused by the onset and progression of kidney diseases, many authors have tried to explain the inflammatory processes that are the baselines of several chronic diseases, as well as the biomarkers that participate in that process. This paper aims to make a narrative literature review on the pathophysiology of the progression of glomerular kidney disease as well as the possible roles played by chemokines as progression markers of renal disease.

KEYWORDS: Biomarkers. Chemokines. Chronic kidney failure.

\section{Introdução}

Os dados epidemiológicos da doença renal crônica (DRC) demonstram um aumento considerável dessa doença em todo mundo. Dados do Estudo Longitudinal de Saúde do Adulto (ELSA)-BRASIL, demonstraram prevalência de 9,9\% de Taxa de Filtração Glomerular diminuída (TFG) $\left(<60 \mathrm{~mL} / \mathrm{min} / 1,73 \mathrm{~m}^{2}\right)$ em adultos brasileiros com diabetes (BARRETO et al., 2016). As últimas diretrizes publicadas pelo Clinical Practice Guideline for the Evaluation and Management of Chronic Kidney Disease, (KDIGO) definem a DRC como a presença de anormalidades da estrutura ou função dos rins, presente por mais de três meses, com implicações para a saúde. Os critérios para diagnóstico incluem a albuminúria e o decréscimo da TFG $<60 \mathrm{~mL} / \mathrm{min} / 1,73 \mathrm{~m}^{2}$ (BARRETO et al., 2016; KDIGO, 2013).

Lesões frequentes e contínuas nos rins poderão culminar na perda definitiva de suas funções, levando a cronicidade da doença devido às agressões que causam danos às células e/ou tecidos e geram uma resposta inflamatória a partir da ativação e estímulo contínuo do sistema imune. As moléculas mediadoras das respostas inflamatórias agem nas células agredidas, levando a graves comprometimentos dos tecidos, muitas vezes de forma crônica e irreversível (MOSS et al., 2004; EL NAHAS; BELLO, 2005).

No caso do comprometimento do tecido renal, a inflamação participa de forma ativa dos mecanismos de pro- gressão da lesão renal em doenças de diversas causas. Neste cenário, se destacam estudos recentes que enfocam a inflamação subclínica na progressão das doenças renais, em especial nas glomerulopatias, e o papel que algumas quimiocinas têm como biomarcadores na progressão da DRC (CHUNG, 2011; SIMOES E SILVA, 2011; TEIXEIRA, 2014).

Visando realizar o diagnóstico precoce da DRC e minimizar os aspectos econômicos e os impactos à vida do paciente causados pela instalação e progressão da doença renal, muitos autores procuram explicar os processos inflamatórios que são as linhas de bases de muitas doenças crônicas e os biomarcadores que participam desse processo. Este artigo tem por objetivo fazer uma revisão de literatura narrativa sobre a fisiopatologia da progressão da doença renal glomerular, bem como, as possíveis funções das quimiocinas como marcadores de progressão da doença renal.

\section{Inflamação na doença renal de acometimento glomerular}

O glomérulo renal é divido em compartimentos que são separados pela membrana basal glomerular (MBG). O compartimento endocapilar é composto pela rede capilar circundada pelas células mesangiais e o compartimento extracapilar é composto pelos podócitos, espaço de Bowman e pela cápsula de Bowman. Em modelos animais, a progressão das doenças glomerulares inicia-se com o envolvimento do compartimento extracapilar, interferindo na barreira de filtra-

DOI: https://doi.org/10.25110/arqsaude.v21i2.2017.5731

${ }^{1}$ Acadêmicos de Medicina - Campus Centro Oeste, Universidade Federal de São João Del-Rei (UFSJ), Minas Gerais/Brasil.

${ }^{2}$ Farmacêutico - Doutorando em Fisiologia e farmacologia no Instituto de Ciências Biológicas da Universidade Federal de Minas Gerais( UFMG)/MG

${ }^{3}$ Enfermeira - Doutora em Ciências da Saúde pelo programa de Infectologia e Medicina Tropical da Faculdade de Medicina da Universidade Federal de Minas Gerais. Professora Adjunta II/ Universidade Federal de São João Del-Rei /UFSJ. Campus Centro Oeste, Universidade Federal de São João Del-Rei, Divinópolis/MG -Brasil. Rua Sebastião Gonçalves Coelho, 400 - Chanadour. CEP: 35501-296. Divinópolis-MG. Email: albaotoni01@yahoo.com.br 
ção e na progressão da doença, ocasionando obliteração do espaço de Bowman por meio da adesão entre o tufo capilar glomerular e a cápsula de Bowman (KRIZ; LEHIR, 2005).

Prioritariamente são descritos três mecanismos responsáveis pela progressão da doença glomerular em direção à perda dos néfrons: 1) mecanismos desreguladores que acontecem por meio da indiferenciação dos podócitos e provoca defeitos regulatórios seguidos por proliferação celular dentro do espaço de Bowman e colapso do tufo glomerular com perda dos capilares; 2) mecanismos degenerativos ocorrem por meio da perda progressiva dos podócitos levando à fixação de células epiteliais parietais à MBG, seguida pelo estabelecimento de adesão entre os tufos glomerulares e a cápsula de Bowman. Assim, a filtração mal orientada na direção do interstício resulta na deposição excessiva de proteínas nos glomérulos; 3 ) mecanismo inflamatório, que será o mais abordado nessa revisão, ocorre por atividade anormal dos podócitos levando à fixação destes na membrana basal parietal, seguido pelo estabelecimento de adesão do tufo glomerular à cápsula de Bowman (KRIZ; LEHIR, 2005; HARRIS; NEILSON, 2006; LIU, 2006).

A inflamação glomerular inicia-se no compartimento extracapilar, afetando os capilares e o mesângio. Até esse ponto, ainda é possível haver restituição da lesão. Entretanto, com sua evolução, o processo endocapilar pode ter um curso agressivo e provocar aberturas na $\mathrm{MBG}$ com exsudação para dentro do espaço de Bowman. Essa quebra na MBG e a disseminação da inflamação ocasionam a formação de crescentes no espaço de Bowman, constituindo uma injúria que provavelmente causará a morte do glomérulo e a perda do néfron. As crescentes são estruturas histológicas formadas em muitas doenças glomerulares. São assim chamadas porque se assemelham à lua na sua fase crescente, mas, na realidade, são caracterizados por proliferação celular extracapilar, infiltração de células inflamatórias e depósito de fibrina e tecido conectivo, levando à obstrução parcial ou total do espaço de Bowman (FOGO, 2007). Essas estruturas têm seu conteúdo formado, principalmente, por células epiteliais derivadas dos podócitos e das células epiteliais parietais; uma matriz intercelular formada por um material amorfo semelhante ao da membrana basal; e macrófagos. Além disso, a inflamação endocapilar induz a ocorrência de alterações morfológicas nos podócitos. Essas alterações têm por característica serem microprojeções espessas e irregulares no compartimento apical do corpo das células. Este fenótipo dos podócitos está associado com a formação de pontes podocitárias entre o tufo glomerular e a cápsula de Bowman. No entanto, o mecanismo celular de formação dessas pontes ainda não é conhecido ((KRIZ; LEHIR, 2005; FOGO, 2007).

A inflamação que se iniciou no glomérulo em razão de alguma agressão sofrida por este, desencadeia diversos mecanismos lesivos por meio da ativação do sistema imunológico de forma contínua e exacerbada, que acabará também por lesar o espaço do túbulo intersticial. Assim, os principais mecanismos que contribuem conjuntamente e para a progressão da lesão renal são: (1) hipertensão glomerular, (2) hiperfiltração glomerular; (3) proteinúria; (4) liberação de citocinas/quimiocinas; (5) acúmulo de macrófagos e linfócitos T; (6) alterações na membrana basal e nas células adjacentes com transformação de células mesenquimais em fibroblastos; (6) síntese de matriz extracelular, com desarranjo estru- tural, disfunção e morte celular no interstício (KRIZ; LEHIR, 2005; HARRIS; NEILSON, 2006; LIU, 2006; FOGO, 2007).

A hipertensão glomerular está estreitamente relacionada ao papel que o sistema renina angiotensina aldosterona (SRAA) exerce no mecanismo de inflamação e consequente evolução para a DRC. Fisiologicamente, a angiotensina II (Ang II) provoca uma maior vasoconstrição da arteríola glomerular eferente em relação à arteríola aferente, provocando o aumento da pressão hidrostática no capilar glomerular. Embora essa resposta seja aparentemente benéfica para manter a homeostase, os estudos sugerem que a elevação persistente da pressão hidrostática no capilar glomerular resulta em proteinúria, glomerulosclerose e perda definitiva de néfrons (LIU, 2006; BRENNER, 2002). Além disso, a Ang II é responsável por mediar efeitos pró-fibróticos como promover a migração de células endoteliais e células musculares lisas dos vasos sanguíneos e promover a hipertrofia e hiperplasia dessas células musculares lisas do tecido vascular e das células mesangiais. Todos os componentes do SRAA estão presentes nos macrófagos, os quais podem servir como outra fonte de Ang II. Esta também induz a produção de outros fatores de crescimento, incluindo fator de crescimento de fibroblasto, fator de crescimento derivado das plaquetas (PDGF) e TGF- $\beta$, além do ativador do inibidor de plasminogênio tipo 1 (PAI-1), sendo que todos estes podem impactar na fibrose. O SRAA é um importante foco de estudo na progressão da DRC em razão da eficácia da inibição desse sistema, por meio da utilização dos inibidores da enzima conversora de angiotensina (IECA) ou bloqueadores do receptor de angiotensina (BRA), no retardo da progressão dessa doença - efeito antifibrótico, antiproteinúrico e de redução da hipertensão sistêmica e intraglomerular (FOGO, 2007).

A hiperfiltração é um mecanismo compensatório realizado pelos glomérulos remanescentes à medida que há progressão da doença renal. Essa tentativa de compensar a perda da função renal pela diminuição do número de néfrons funcionantes, no entanto, provoca uma perda da autorregulação, o que agrava a hipertensão intraglomerular. Como resultado observa-se diminuição da seletividade da membrana de filtração glomerular e, consequentemente, o surgimento de proteinúria não seletiva, bastante comum na nefropatia diabética (HARRIS; NEILSON, 2006; FOGO, 2007; ZOJA; ABBATE; REMUZI, 2006).

No processo de proteinúria, que se originou em decorrência da diminuição da seletividade da membrana de filtração glomerular pelas agressões sofridas, parte das proteínas filtradas em excesso pelo glomérulo é reabsorvida nos túbulos proximais por endocitose. Isso leva a uma série de respostas prejudiciais nas células tubulares, incluindo a apoptose, síntese de componente do sistema do complemento e expressão de mediadores vasoativos e fibrogênicos (MORENO, 2014). Além disso, os depósitos de material proteináceo nas células tubulares proximais promovem edema e ruptura dos lisossomos intracelulares, lesão tubular direta e estresse oxidativo (ALFREY, 1992). A albuminúria, em especial, ativa as proteínas do complemento (NANGAKU; PIPPIN; COUSER, 2002) os fatores nucleares $\kappa-\beta(N F k-\beta)$ e estimula a liberação de mediadores inflamatórios em especial as quimiocinas e fatores de crescimento, tais como fator de crescimento semelhante à insulina tipo 1 (IGF-1) e TGF- $\beta$. Além disso, a proteinúria também promove o recrutamento 
de macrófagos, que perpetuam a reação inflamatória intersticial, produzindo a nefrite intersticial (WANG; LAPAGE; HIRSCHBERG, 2002).

Todo o processo de lesão renal, incluindo a proteinúria e sua reabsorção pelos túbulos proximais, faz com que estas células liberem citocinas/quimiocinas que atuarão recrutando células do sistema imunológico para o sítio de inflamação renal.

As células tubulares reagem a este processo inflamatório pela transição epitelial-mesenquimal glomerular e tubular, mediada pelas citocinas, se transformando em fibroblastos intersticiais, além de promover o desarranjo da membrana basal pelas proteases originadas de células epiteliais afetadas do glomérulo. Proteínas da superfamília do TGF- $\beta$, particularmente o TGF- $\beta$ e a proteína morfogenética óssea (BMP-7), representam os principais mediadores desse processo. Enquanto o TGF- $\beta$ estimula a transição epitelial-mesenquimal e a síntese de proteínas da matriz extracelular, como colágenos e fibronectina, a BMP-7 inibe a transição epitelial-mesenquimal e estimula a síntese de enzimas proteolíticas, como as metaloproteinases MMP-2 e MMP-9 (HARRIS; NEILSON, 2006; LIU, 2006; FOGO, 2007). Outros agentes biológicos podem também modular a ligação do TGF- $\beta$ ao seu receptor, incluindo o fator de crescimento do tecido conjuntivo (ABREU; KETPURA, 2002).

Dessa forma, as alterações dos glomérulos e túbulos refletem no interstício, levando a formação de uma cicatriz túbulo-intersticial que é o resultado final do processo inflamatório, sendo composta principalmente de fibronectina, e colágenos tipo I e tipo III, além de outras glicoproteínas, como a trombospondina, a osteopontina e os proteoglicanos (HARRIS; NEILSON, 2006; LIU, 2006; FOGO, 2007; KELLY; NEILSON, 2004). Os fibroblastos formados a partir da transição epitelial-mesenquimal do glomérulo, podem proliferar-se, migrar para áreas adjacentes ao processo inflamatório e, então, promover a síntese e deposição de fibronectina e colágeno (STRUTZ; NEILSON, 2003). Nas fases iniciais do processo, predomina o colágeno tipo III. À medida que o processo fibrogênico progride, há substituição do colágeno tipo III pelo colágeno tipo I. Os fibroblastos túbulo-intersticiais podem secretar colágenos tipo I, III, IV e V, principalmente em resposta à ação do TGF- $\beta$. A fase final do processo culmina em apoptose dos fibroblastos e formação da cicatriz acelular. Portanto, a fibrose renal, caracterizada pela glomeruloesclerose e fibrose túbulo-intersticial, é a via final comum da DRC (KELLY; NEILSON, 2004; HARRIS; NEILSON, 2006; LIU, 2006; FOGO, 2007).

Salienta-se que a progressão da DRC tende a evoluir para eventos comuns independentemente do processo patológico de base e dos fatores de risco específicos secundários. Esses eventos são caracterizados pela fibrose glomerular progressiva, perda de capilaridade peritubular, causando hipóxia tubular e destruição dos néfrons funcionantes em razão de atrofia tubular. Esses mecanismos complexos resultam fatalmente na perda das funções renais (HARRIS; NEILSON, 2006; LIU, 2006; FOGO, 2007).

\section{As quimiocinas como biomarcadores da lesão renal}

As quimiocinas pertencem à família das citocinas quimiotáticas que foram primeiramente identificadas com base em sua habilidade em induzir a migração de diferentes tipos de células, particularmente células de origem linfoide, em especial em situação de resposta imunológica em processos de agressão tecidual. Caracterizam-se por serem proteínas solúveis de baixo peso molecular, variando entre 8 a 17 $\mathrm{kDa}$. Além da quimiotaxia possuem a função de ativação dos leucócitos e são capazes de influenciar a hematopoese e de modular a angiogênese (STRUTZ; NEISON, 2003; SIMÕES E SILVA et al., 2011; CHUNG; LAN, 2011). Atualmente, são conhecidas mais de 50 quimiocinas humanas, as quais estão classificadas estruturalmente em quatro classes: $\mathrm{CXC}, \mathrm{CC}$, C e CX3C (MORENO et al., 2014; TEIXEIRA et al., 2007).

No desenvolvimento da injúria renal crônica, as quimiocinas e seus receptores desempenham um papel crítico no recrutamento de células T, macrófagos e células dendríticas (CHUNG; LAN, 2011; HOLDSWORTH, 2007). Alguns autores admitem que a alteração no perfil de citocinas, em especial, as quimiocinas em pacientes com lesão renal progressiva, de causas variadas, pode contribuir para o surgimento da proteinúria e posterior lesão glomerular de amplo espectro (ARAYA et al., 2006; VAYADA et al., 2011).

A IL-8/CXCL8, primeira quimiocina a ser descoberta, exerce predominantemente o papel de efeito quimioatraente para neutrófilos e ativação da proteinúria associada com a doença renal (SIMÕES E SILVA et al., 2011). Ao que parece esta quimiocina atua de forma importante tanto nas fases agudas, quando seus níveis urinários mostraram-se elevados em pacientes na fase aguda de várias formas de glomerulonefrites (SEGERER; SCHLONDORFF, 2007; SEGERER et al., 2006) quanto na fase crônica sendo também identificado um número aumentado de CXCR1-neutrófilos positivos (receptor da IL-8/CXCL8) nos glomérulos e na região túbulo-intersticial dos pacientes com glomerulonefrite membranosa proliferativa, nefrite lúpica e glomerulonefrite crescente, ou mesmo identificado níveis urinários aumentados nas fases avançadas de nefropatia por IgA como descrito por Huang et al em 2001 (CHUNG; LAN, 2011; HUANG et al., 2001). No entanto, um estudo publicado por Li et al em 2006, identificou níveis aumentados de IL-8/CXCL8 em pacientes com lúpus, porém, não observou diferença significativa entre pacientes com e sem comprometimento renal (LI et al., 2006).

A quimiocina RANTES/CCL5 também conhecida como RANTES, bem como seus receptores têm sido utilizados como marcadores de lesões renais evidenciadas pela proteinúria. Em estudo de Segerer et al. (2006) foi identificado que na nefrite intersticial aguda, embora a indução da produção da RANTES/CCL5 seja mais tardia quando comparada a MCP-1/CCL2, permanece com níveis elevados por mais dias na corrente sanguínea (SEGERER et al., 2006). Outro estudo mostrou o aumento dos níveis séricos de MCP-1/CCL2 e RANTES/CCL5 em pacientes com nefropatia diabética que apresentavam hiperglicemia com aumento da produção de espécies reativas de oxigênio (ROS) (ELMARAKBY; SULLIVAN, 2012). Em estudo experimental, camundongos com proteinúria devido à indução de nefrite nefrotóxica aumentaram a expressão glomerular de MCP-1/CCL2, RANTES/CCL5 e IL-10/CXCL10 (SCHADDE, 2000).

A MCP-1/CCL2 vem sendo citada como importante mediadora dos processos renais. Estudos mostraram grande quantidade da MCP-1/CCL2 em tecidos renais de pacientes 
portadores de doenças glomerulares progressivas, rejeições a transplantes e nefrites intersticiais acarretando infiltração de macrófagos na patogênese da renal (KIM; TAM, 2011; SELLARES et al 2013). Como já descrito anteriormente, praticamente todos os tipos de células renais intrínsecas (endotelial, mesangiais, células epiteliais tubulares e podócitos) são capazes de produzir MCP-1/CCL2 quando esse tecido é lesado (KIM; TAM, 2011). Ho e colaboradores identificaram que os casos de perda tardia (após seis meses) do transplante renal estavam associados com elevada taxa de MCP-1/CCL2 urinária quando comparada a enxertos com função renal preservada (HO et al., 2013). Níveis elevados desta quimiocina já haviam sido relatados anteriormente em pacientes com rejeição aguda do enxerto renal, além da constatação da redução significativa desta quimiocina nos pacientes que responderam ao tratamento anti-rejeição (DUBI'NSKI et al., 2008).

Estudos relevantes sobre as citocinas na doença renal crônica estão resumidos na tabela 1 .

Tabela 1: Resumo de estudos relevantes sobre as citocinas e quimiocinas da doença renal crônica

\begin{tabular}{|c|c|c|c|}
\hline Ref & Modelo & Citocina & Principais Resultados \\
\hline 43 & Humano $(\mathrm{N}=30$ adultos $)$ & MCP-1/CCL2 & $\begin{array}{l}\text { O indicador mais importante de doença renal estágio terminal na nefropatia } \\
\text { idiopática membranosa encontrado neste estudo foi um infiltrado intersticial } \\
\text { de células CD68-positivas acompanhado da expressão de MCP-1/CCL2/ } \\
\text { CCR2. }\end{array}$ \\
\hline 41 & Humano $(\mathrm{N}=71$ crianças $)$ & MCP-1/CCL2 & $\begin{array}{l}\text { Os níveis urinários de MCP } 1 / \text { CCL2 apresentaram-se aumentados e } \\
\text { correlacionados com proteinúria em crianças com Nefropatia por Ig A e } \\
\text { Glomeruloesclerose Segmentar e Focal. }\end{array}$ \\
\hline 21 & Humano $(\mathrm{N}=70$ adultos $)$ & MCP-1/CCL2 & $\begin{array}{l}\text { O estudo mostrou associação entre a expressão do receptor Toll-like- } 4 \text { com } \\
\text { o marcador pró-inflamatório MCP-1/CCL2 em biópsias de rins de pacientes } \\
\text { com DRC, sugerindo que o aumento da expressão desta é uma característica } \\
\text { importante da DRC. }\end{array}$ \\
\hline 8 & Humano $(\mathrm{N}=96$ adultos $)$ & MCP-1/CCL2 & $\begin{array}{l}\text { Os níveis urinários de } \mathrm{MCP}-1 / \mathrm{CCL} 2 \text { correlacionaram-se positivamente } \\
\text { com o envolvimento renal do lúpus eritematoso sistêmico com razoável } \\
\text { sensibilidade, especificidade e valores preditivos para detectar a nefrite lúpica. }\end{array}$ \\
\hline 44 & Animal & RANTES/CCL5 & $\begin{array}{l}\text { A quimiocina RANTES/CCL5 produzida pelas células tubulares renais tem } \\
\text { papel fundamental no desenvolvimento de DRC. }\end{array}$ \\
\hline 42 & Animal & RANTES/CCL5 & $\begin{array}{l}\text { A RANTES pode ser um potencial alvo terapêutico nas nefrites túbulo- } \\
\text { intersticial. Neste estudo a menor expressão renal de RANTES atenua a nefrite } \\
\text { imuno-mediada, em razão dos ratos terem desenvolvido significativamente } \\
\text { menos proteinúria, azotemia, e inflamação renal, com reduzida formação de } \\
\text { crescentes e de nefrite túbulo-intersticial. }\end{array}$ \\
\hline 38 & Humano ( $\mathrm{N}=73$ adultos) & RANTES/CCL5 & $\begin{array}{l}\text { O estudo observou que o aumento persistente dos níveis urinários de } \\
\text { RANTES, após remissão inicial, é um preditor de recaída renal em pacientes } \\
\text { com glomerulonefrite proliferativa difusa por lúpus eritematoso sistêmico. }\end{array}$ \\
\hline 36 & In Vitro & IL-8/CXCL8 & $\begin{array}{l}\text { Identificou-se que a albumina é um forte estímulo para a expressão tubular } \\
\text { de IL-8. }\end{array}$ \\
\hline 27 & Humano $(\mathrm{N}=16$ adultos $)$ & IL-8/CXCL8 & $\begin{array}{l}\text { Em pacientes hipertensos com doença renal crônica o uso do bloqueador do } \\
\text { receptor de angiotensina Irbesartan ofereceu renoproteção adicional de uma } \\
\text { forma dependente da dose, reduzindo a excreção de citocinas pró-inflamatórias } \\
\text { na urina de pacientes com DRC, entre elas a CXCL8. }\end{array}$ \\
\hline 18 & Humano ( $\mathrm{N}=40$ adultos) & IL-8/CXCL8 & $\begin{array}{l}\text { Observou-se que a suplementação de cúrcuma em pacientes com nefropatia } \\
\text { diabetes tipo } 2 \text { favoreceu a diminuição da proteinúria e dos níveis séricos e } \\
\text { urinários de IL-8 entre os valores pré e pós suplementação de cúrcuma, em } \\
\text { pacientes com nefropatia por diabetes tipo } 2 \text {. Sugeriu-se que a suplementação } \\
\text { de cúrcuma auxiliou-se na melhora da progressão da doença renal. }\end{array}$ \\
\hline
\end{tabular}

\section{Conclusão}

As evidências a partir de estudos clínicos demonstram que as quimiocinas desempenham importante papel nos mecanismos de acometimento renal de várias doenças renais seja agudo ou crônico. Algumas, em especial a MCP-1/ CCL2, têm um grande potencial como um biomarcador para o diagnóstico, prognóstico e resposta à terapia. Contudo, talvez pelos custos elevados, os estudos têm apresentado números reduzidos de pacientes, fato que limita a ampliação da aplicação dos resultados na prática clínica.

\section{Referências}

ABREU, J. G. et al. Connective-tissue growth factor (CTGF) modulates cell signaling by BMP and TGF- $\beta$. Nat Cell Biol, 4:599-604, 2002

ALFREY, A. C. Toxicity of tubule fluid iron in the nephrotic syndrome. Am J Physiol,; 263(4):F637-41, 1992

ARAYA, C. E. et al. A case of unfulfilled expectations: Cytokines in idiopathic minimal lesion nephrotic syndrome. Pediatr Nephrol, 21(5):603-10, 2006. 
BARRETO, S. M. et al. Chronic kidney disease among adult participants of the ELSA-Brasil cohort: association with race and socioeconomic position. J Epidemiol Community Health, 70(4):380-9, 2016

BRENNER, B. M. Remission of renal disease: recounting the challenge, acquiring the goal. J Clin Invest, 110(12):1753-8, 2002

CHUNG, A. C; LAN, H. Y. Chemokines in renal injury. J Am Soc Nephrol, 22:802-9, 2011.

DUBI'NSKI, B. et al. "Activated cells in urine and monocyte chemotactic peptide-1 (MCP-1) - sensitive rejection markers in renal graft recipients," Transplant Immunology, 18(3):203- 207, 2008.

EL NAHAS, A. M.; BELLO, A. K. Chronic kidney disease: the global challenge. Lancet, 365(9456):331-40, 2005.

ELMARAKBY, A. A; SULLIVAN, J. C. Relationship between oxidative stress and inflammatory cytokines in diabetic nephropathy. Card Therpeutics, 30:49-59, 2012.

EL-SHEHABY, A. et al. Correlations of urinary biomarkers, TNF-like weak inducer of apoptosis (TWEAK), osteoprotegerin (OPG), monocyte chemoattractant protein-1 (MCP-1), and IL-8 with lupus nephritis. Journal of Clinical Immunology, 31:848-56, 2011

FOGO, A. B. Mechanisms of progression of chronic kidney disease. Pediatr Nephrol, 22(12):2011-22, 2007.

HARRIS, R. C.; NEILSON, E. G. Toward a unified theory of renal progression. Annu Rev Med, 57:365-80, 2006.

$\mathrm{HO}$, J. et al. "Increased urinary CCL2: Cr ratio at 6 months is associated with late renal allograft loss," Transplantation, 95(4): 595-602, 2013allograft loss," Transplantation, 95(4): 595-602, 2013.

HOLDSWORTH, S. R. Tipping PG. Leukocytes in glomerular injury. Semin Immunopathol, 29:355-74, 2007.

HUANG, F. et al. Urinary levels of interleukin-8 (IL-8) and disease activity in patients with IgA nephropathy. J Clin Lab Anal, 15:30-4, 2001.

International Society of Nephrology. Clinical Practice Guideline for the Evaluation and Management of Chronic Kidney Disease. Kidney Int Suppl, 3:1-150, 2013.

KELLY, C. J; NEILSON, E. G. TUBULOINTERSTITIAL DISEASES. IN THE KIDNEY. 2004; 1483-512.

KHAJEHDEHI, P. et al. ORAL SUPPLEMENTATION OF TURMERIC attenuates proteinuria, transforming growth factor-beta and interleukin-8 levels in patients with overt type 2 diabetic nephropathy: a randomized, double-blind and placebo-controlled study. Scandinavian Journal of Urology and Nephrology, 45:365-370, 2011.
KIM, M. J.; TAM, F. W. K. "Urinary monocyte chemoattractant protein-1 in renal disease," Clinica Chimica Acta, 412, 23-24: 2022-2030, 2011.

KRIZ, W.; LEHIR, M.; Pathways to nephron loss starting from glomerular diseases-insights from animal models. Kidney Int, 67:404-419, 2005.

LEPENIES, J. et al. Renal TLR4 mRNA expression correlates with inflammatory marker MCP-1 and profibrotic molecule TGF- $\beta(1)$ in patients with chronic kidney disease. Nephron Clinical Practice, 119:97-104, 2011.

LI, Y. et al. Urinary biomarkers in lupus nephritis. Autoimmun Rev, 5:383-8, 2006.

LIU, Y. Renal fibrosis: New insights into the pathogenesis and therapeutics. Kidney Int, 69(2):213-7, 2006.

MORENO, J. A.et al. Role of chemokines in proteinuric kidney disorders. Expert Rev Mol Med, 16:e3, 1-21, 2014.

MOSS, R. B. et al. Th1/Th2 cells in inflammatory disease states: therapeutic implications. Expert Opin Biol Ther, 4(12):1887-96, 2004

NANGAKU, M.; PIPPIN, J.; COUSER, W. G. C6 mediates chronic progression of tubulointerstitial damage in rats with remnant kidneys. J Am Soc Nephrol 2002; 13:928-36.

NI, J.; HUANG, H. Q.; LÜ, L. L.; ZHENG, M.; LIU, B. C. Influence of irbesartan on the urinary excretion of cytokines in patients with chronic kidney disease. Chinese Medical Journal (Engl.), 125:1147-1152, 2012.

PECOITS-FILHO R.; SYLVESTRE, L. C.; STENVINKEL, P. Chronic kidney disease and inflammation in pediatric patients: from bench to playground. Pediatr Nephrol, 20(6):714-20, 2005.

SCHADDE, E. Expression of chemokines and their receptors in nephrotoxic serum nephritis. Nephrology Dialysis Transplantation, 15:1046-1053, 2000.

SEGERER, S. et al. Expression of the chemokine receptor CXCR1 in human glomerular diseases. Kidney Int, 69:1765-73, 2006.

SEGERER, S; SCHLONDORFF, D. Role of chemokinesfor the localization of leukocyte subsets in the kidney. Semin Nephrol, 27:260-74, 2007.

SELLARES, J. et al. "Molecular diagnosis of antibodymediated rejection in human kidney transplants," American Journal of Transplantation, 13(4):971-83, 2013.

SIMÕES e SILVA. et al. CMBM, Vianna HR. Inflamação na doença renal crônica: papel de citocinas. J Bras Nefrol, 33(3):351-64, 2011.

STENVINKEL, P. et al. IL-10, IL-6, and TNFalpha: central 
factors in the altered cytokine network of uremia - the good, the bad, and the ugly. Kidney Int, 67(4):1216-33, 2005.

STRUTZ, F.; NEILSON, E. G. New insights into mechanisms of fibrosis in immune renal injury. Springer Semin Immuno pathol, 24:459-76, 2003.

TANG, S. et al. Albumin stimulates interleukin-8 expression in proximal tubular epithelial cells in vitro and in vivo. Journal of Clinical Investigation, 111:515-527, 2003.

TEIXEIRA, A. L. et al. Chemokines as Potential Markers in Pediatric Renal Diseases. Dis Markers, 1-9, 2014.

TIAN, S. et al. Urinary levels of RANTES and M-CSF are predictors of lupus nephritis flare. Inflammation Research, 56:304-310, 2007.

VAIDYA, V. S. et al. Regression of microalbuminuria in type 1 diabetes is associated with lower levels of urinary tubular injury biomarkers, kidney injury molecule-1, and $N$-acetyl- $\beta$-D-glucosaminidase. Kidney international, 79(4):464-70, 2011.

WANG, S. N.; LAPAGE, J.; HIRSCHBERG, R. Hirschberg $\mathrm{R}$. Loss of tubular bone morphogenetic protein-7 in diabetic nephropathy. J Am Soc Nephrol, 12:2392-99, 2001.

WASILEWSKA, A. et al. Urinary monocyte chemoattractant protein-1 excretion in children with glomerular proteinuria. Scandinavian Journal of Urology and Nephrology, 45:52-59, 2011.

XIE, C. et al. RANTES deficiency attenuates autoantibodyinduced glomerulonephritis. Journal of Clinical Immunology, 31:128-135, 2011.

YOSHIMOTO, K. et al. CD68 and MCP-1/CCR2 expression of initial biopsies reflect the outcomes of membranous nephropathy. Nephron Clinical Practice, 98:25-34, 2004.

YU, T. M. et al. RANTES mediates kidney ischemia reperfusion injury through a possible role of HIF-1 $\alpha$ and LncRNA PRINS. Sci Rep, 4(6):18-24, 2016.

ZOJA, C.; ABBATE, M.; REMUZZI, G. Progression of chronic kidney disease: insights from animal models. Curr Opin Nephrol Hypertens, 15(3):250-7, 2006. 